\title{
MicroRNAs in CNS injury: Potential roles and therapeutic implications
}

\author{
Sindhu K. Madathil ${ }^{1}$, Peter T. Nelson ${ }^{2,}{ }^{*}$, Kathryn E. Saatman ${ }^{1}$, and Bernard R. Wilfred ${ }^{2}$ \\ ${ }^{1}$ Spinal Cord and Brain Injury Research Center, University of Kentucky Medical Center, University \\ of Kentucky, Lexington, KY, USA \\ ${ }^{2}$ Department of Pathology, Division of Neuropathology, University of Kentucky Medical Center \\ and Sanders-Brown Center on Aging, University of Kentucky, Lexington, KY, USA
}

\author{
Keywords \\ Alzheimer's; miR-107; SCI; stroke; TBI; trauma
}

\section{Introduction}

Central nervous system (CNS) injuries, such as cerebral ischemia, traumatic brain injury (TBI), and spinal cord injury (SCI), are major causes of death and disability in the adult population [1-3]. Although the etiologies of stroke and traumatic injury are different, they share many common pathological mechanisms. The early phase of stroke and trauma is characterized by disruption of the blood-brain barrier, reduced or altered blood flow, and neuronal and glial damage [4]. Secondary injury evolves from this initial damage over a period of days to weeks and even months, marked by a complex series of events including altered calcium homeostasis, free radical generation, oxidative stress, inflammation, necrosis, apoptosis, and/or axonal injury, culminating in degeneration of cells and synapses [4].

MicroRNAs (miRNAs) are 22 nucleotide RNAs that are a subset of small non-coding RNAs [5]. The main functions of miRNAs are thought to relate to binding to "target" mRNA through partial sequence complementarity and regulating (generally inhibiting) gene expression at the level of mRNA translation. MiRNAs are abundantly expressed in the mammalian CNS, where they control complex processes associated with neuronal differentiation and maturation and are likely to be important mediators of neuronal function [6,7]. Compared to other CNS pathologies [8,9], much less is known about the miRNA expression changes seen in CNS injury. However, as we hypothesize below, miRNA changes that relate directly to acute CNS injury may also contribute to more chronic brain diseases.

\section{Alterations in, and manipulation of, miRNAs in CNS ischemia}

MiRNA profiling of experimental stroke brains has revealed alterations in many individual miRNAs [10-14] (Table 1). The first miRNA screening study in ischemic rats reported alterations in several miRNAs in the brain at 24 and 48 hours after reperfusion [14]. To correlate the miRNA alterations to their respective targets, a DNA microarray using

(C) 2010 WILEY Periodicals, Inc.

*Corresponding author: Peter T. Nelson, pnels2@email.uky.edu. 
ischemic samples was performed. The mRNA expression of genes encoding visinin-like 1, a neuronal calcium sensor protein, and aquaporins were decreased, whereas their regulatory miRNAs (miR-124a, miR-290, and miR-494) increased in the ischemic cortex. Upregulation of MMP9 was correlated with decreased expression of its regulating miRNAs, miR-132 and miR-664. As aquaporins and MMP9 are involved in stroke pathophysiology [15,16], identification of their regulators may reveal new directions for stroke research. A more detailed temporal miRNA profiling of ischemic rat cortex indicated 8 miRNAs with increased, and 12 with decreased, expression at four out of five reperfusion time points studied between 3 hours and 3 days [12]. Bioinformatics analysis links these miRNAs to several mRNAs known to mediate inflammation, neuroprotection, receptor functions, and ionic homeostasis.

Ischemic preconditioning is a sublethal duration of ischemia that protects the brain against a subsequent severe ischemic insult. MiRNA profiling in the cortex showed significant alterations in 273 miRNAs in preconditioned, 144 miRNAs in ischemic, and 50 miRNAs in tolerant (preconditioned with subsequent ischemia) mice [11]. Methyl-CpG binding protein $(\mathrm{MeCP} 2)$, a transcriptional regulator, was identified as a prominent target for miRNAs altered by preconditioning. In the preconditioned cortex, $\mathrm{MeCP} 2$ protein expression increased concurrently with decreased miR-132, a regulator of MeCP2 expression. Further studies are required to understand how $\mathrm{MeCP} 2$ contributes to ischemic tolerance.

There are early indications that miRNAs have therapeutic potential for stroke patients, highlighting the translational possibilities of miRNAs in CNS injuries. AntagomiR-mediated suppression of miR-145 in the post-ischemic rat brain increased expression of the antioxidant enzyme superoxide dismutase-2 and reduced infarct size [12]. Following ischemia, miR-497 appears to regulate neuronal death by targeting anti-apoptotic genes such as bcl-2 and bcl-w [17]. In addition, antagomiR administration into the cerebral ventricles, to suppress miR-497 expression, leads to attenuated ischemic infarction.

In a related context, ischemia in the retina alters miRNA levels, specifically downregulating miRNAs regulating neo-vascularization [18]. Intraocular injection of precursor RNA (predicted to affect miRNA targets) for miR-31, miR-150, or miR-184 significantly reduced neo-vascularization in a model of ischemic retinopathy, further supporting the possibility of miRNA-based medicine for ischemia.

These potential therapies are not the only possible CNS clinical application related to miRNAs and stroke. MiRNA expression profiles in the blood may be useful as a diagnostic tool for CNS injuries. Animal models of stroke, intracerebral hemorrhage, and kainateinduced seizures revealed certain miRNA alterations were injury-specific, and aspects of brain miRNA profiles were mimicked in blood $[10,14]$, indicating that blood miRNAs may be useful as biomarkers for brain damage [10]. In a recent clinical study [19], blood miRNAs from stroke patients exhibited differential expression profiles as compared to normal controls even several months after stroke onset.

\section{miRNAs modulated in TBI}

As with miRNA changes associated with cerebral infarction, the specific miRNA expression changes associated with TBI are only beginning to be understood. In the mouse hippocampus, after brain injury induced by controlled cortical impact (CCI) [20], microarray analyses established that expression levels were decreased for $\sim 50$ miRNAs, but increased for 35 miRNAs, at acute time points. Real-time PCR performed on a subset of miRNAs confirmed the microarray analysis (Table 1). Predicted targets of these dysregulated miRNAs participate disproportionately in gene pathways (including cell differentiation and morphogenesis) that are involved in TBI pathophysiology [20]. Results from in situ 
hybridization showed a reduction in the expression of miR-107 in ipsilateral hippocampal regions vulnerable to cell death in this model. MiRNA profiling after lateral fluid percussion injury in rats has revealed additional clues about miRNA expression changes after TBI [21]. Microarray analyses of injured cortex at several acute time points (6-72 h) showed alterations in many miRNAs, with a persistent upregulation of miR-21. MiR-21 is an astrocyte-concentrated miRNA that negatively regulates astrocyte cell size and GFAP expression [22]. As opposed to hyperplasia that leads to glial scar formation, acute hypertrophy of astrocytes may be beneficial for wound repair [23]. Strategies aimed at downregulating miR-21 may promote astrocytic hypertrophy following trauma.

We recently confirmed that miR-107 expression is markedly decreased 24 hours after CCI brain injury in mice [24]. Injury-induced downregulation of miR-107 appeared selective, as other brain-enriched miRNAs, including miR-124, did not show a pronounced effect (Fig. 1). Using studies in cultured cells combined with RNA co-immunoprecipitation with downstream microarray (RIP-Chip) assay, miR-107 was shown to strongly target progranulin (PGRN or GRN). Following CCI brain injury, surviving hippocampal neurons showed decreased miR-107 with augmented neuronal GRN expression. GRN protein is involved in wound healing, cell proliferation, and other cellular and biochemical processes [25]. Ongoing work will provide an improved understanding of the roles played by miR-107 and GRN in TBI.

Recent studies also link changes in brain miRNA expression after TBI to the regulation of angiogenesis. For example, miR-107 may participate in angiogenesis through targeting of hypoxia-inducible factor-1 beta and p53 [26]. MiR-320 is also implicated in angiogenesis, where it may impair angiogenesis by downregulating insulin like growth factor-1 protein expression [27]. While miR-320 is downregulated in stroke patients with favorable outcome [19], we observed elevated miR-320 expression in blood vessels near the contusion periphery at 24 hours after CCI brain injury in mice (Fig. 1). If this acute increase in miR-320 modifies post-traumatic angiogenesis, then altering miR-320 expression may offer a novel context to boost adaptive neovascularization.

Despite these suggestive early studies, there is no firmly established "function" for miRNA expression changes in TBI. Future studies using miRNA knockout animals, or delivery of miRNA inhibitors or miRNA itself, may yield insights into the functional aspects of these alterations; however, since one miRNA targets multiple mRNAs, a clearer understanding of miRNA targets through in vitro studies is necessary to design therapeutic interventions. Furthermore, considering that temporal and regional patterns of pathological events vary in different species and experimental paradigms, additional miRNA screening studies are necessary in multiple animal models [28-30].

Although blood miRNAs are known to be altered in patients with ischemic stroke or cerebral hemorrhage [10,19], no reports are currently available regarding miRNA profiles from brain-injured patients. Identification of serum biomarkers is an emerging area of research [31], and miRNA profiles may have utility as biomarkers for TBI. Moreover, miRNA screening from different states of injury severity may provide unique miRNA "fingerprints" that will be particularly helpful in TBI classification.

\section{miRNA changes after SCI}

There have been few studies published about SCI-induced miRNA expression changes. Following contusive SCI in rats, levels of miRNAs that target mRNAs involved in inflammation, oxidative stress, and apoptosis are altered [32]. Using a microarray platform and RTq-PCR, Liu et al. [32] classified alterations in miRNAs into three categories: (1) upregulated, (2) downregulated, and (3) early upregulation at 4 hours followed by 
downregulation at 1 and 7 days after SCI. In a model of spinal cord compression, microarray profiling identified 10 differentially expressed miRNAs compared to controls, 12 hours after injury in mice [33]. Subsequent RTq-PCR revealed increased miR-223 expression at 6 hours, 12 hours and 3 days, and decreased miR-124 expression from 1 to 7 days after SCI. In situ hybridization results demonstrated cellular expression of miR-223, a myeloid-specific miRNA, and absence of miR-124 around the compression injury site. The functional implications of these results remain to be elucidated, but these studies provide an early indication that miRNA dysregulation is a feature of SCI.

\section{Acute CNS injury and neurodegenerative diseases: Possible links between pathogenetic pathways?}

Although distinct in many ways, stroke, TBI, and SCI have common pathological mechanisms and are reported as risk factors for sporadic neurodegenerative diseases [34,35]. Approximately $40 \%$ of the risk for Alzheimer's disease (AD) is thought to be derived from environmental risk factors [36], the identities of which are largely unknown. Extensive evidence (some circumstantial) indicates that acute CNS injuries may contribute to neurodegenerative diseases [34,35,37,38]. For example, increased amyloid precursor protein (APP) immunoreactivity is seen within injured axons [39], which is interesting, considering that overexpression of the APP gene (such as in Down syndrome or more focal APP duplication) induces AD-type dementia [40]. CNS injuries including stroke and TBI are also reported as precursors for Parkinson's disease, amyotrophic lateral sclerosis (ALS), and frontotemporal lobar dementia (FTLD) [34,37,41-44]. These associations suggest that there are currently under-appreciated biological mechanisms linking acute brain injury and neurodegenerative diseases.

A number of miRNAs altered in CNS injury show similar dysregulation in neurodegenerative diseases, compatible with the hypothesis that miRNAs may help interweave acute CNS injury and chronic neurodegeneration (Fig. 2). MiRNA changes following trauma may have the potential to induce or accelerate the progression of chronic neurodegeneration. Conversely, miRNAs altered in neurodegenerative diseases may have roles or reactive functions related to how well cells cope following other insults such as trauma and ischemia.

In the CCI model of TBI, we and others have observed decreases in miR-107 [20,24], a miRNA which also exhibits expression changes in $\mathrm{AD}, \mathrm{SCI}$, ischemia, and hypoxic insult $[14,26,32,45,46]$. We have previously shown that miR-107 targets beta-site amyloid precursor protein-cleaving enzyme 1 (BACE1) and thus a reduction in miR-107 can accelerate Alzheimer's-type pathology through increased BACE activity [45]. In animal models of TBI and in brain-injured humans, increased BACE1 mRNA expression or immunoreactivity has been associated with accumulation of $A \beta$ peptides $[47,48]$. A downregulation in miR-107 expression in the injured cortex and hippocampus after cortical impact may also lead to increased BACE1 and subsequent $\mathrm{A} \beta$ production, illustrating a potential pathogenetic link between brain injury and AD.

Another mechanism leading to neurodegeneration after acute CNS injury could be chronic inflammation. MiRNAs involved in inflammation are altered after CNS insults. For example, miR-132 was found to be decreased in ischemic cortex. This miRNA is known to potentiate anti-inflammatory signaling [49] and thus a reduction in miR-132 could accelerate inflammation leading to neurodegeneration. 
Another common link is miR-145, a miRNA recently implicated in positively regulating apoptosis [50] which is upregulated following acute CNS injury. Apoptosis is a cell death mechanism with possible relevance to neurodegenerative diseases.

This combined evidence leads us to predict that miRNA alterations following acute CNS injuries will act as a contributing cofactor in the development of "idiopathic" neurodegeneration (Fig. 2).

In summary, cellular injuries in TBI, SCI, and ischemia have overlapping response mechanisms that include miRNA expression changes. In the future, we may better understand the "cross-talk" between different mechanisms and how they relate to neurodegeneration or neuroprotection. The fact that manipulation of one miRNA affects many genes makes miRNAs attractive targets for therapeutic intervention. We anticipate that a better understanding of the miRNAs involved in CNS injuries will open up new therapeutic possibilities.

\section{Abbreviations}

$\begin{array}{ll}\text { AD } & \text { Alzheimer's disease } \\ \text { APP } & \text { amyloid precursor protein } \\ \text { BACE } & \text { beta-site amyloid precursor protein-cleaving enzyme } \\ \text { CCI } & \text { controlled cortical impact } \\ \text { CNS } & \text { central nervous system } \\ \text { miRNA } & \text { microRNA } \\ \text { PGRN/GRN } & \text { progranulin } \\ \text { SCI } & \text { spinal cord injury } \\ \text { TBI } & \text { traumatic brain injury }\end{array}$

\section{Acknowledgments}

We apologize for papers not cited due to space limitations. This study was supported by NIH grants R01 NS061933, K08 NS050110, and P01 NS058484, and Kentucky Spinal Cord and Head Injury Research Trust grant $7-20$.

\section{References}

1. Wyndaele M, Wyndaele JJ. Incidence, prevalence and epidemiology of spinal cord injury: what learns a worldwide literature survey? Spinal Cord. 2006; 44:523-529. [PubMed: 16389270]

2. Feigin VL. Stroke epidemiology in the developing world. Lancet. 2005; 365:2160-2161. [PubMed: 15978910]

3. Javouhey E, Guerin AC, Chiron M. Incidence and risk factors of severe traumatic brain injury resulting from road accidents: a population-based study. Accid Anal Prev. 2006; 38:225-233. [PubMed: 16242108]

4. Bramlett HM, Dietrich WD. Pathophysiology of cerebral ischemia and brain trauma: similarities and differences. J Cereb Blood Flow Metab. 2004; 24:133-150. [PubMed: 14747740]

5. Filipowicz W, Bhattacharyya SN, Sonenberg N. Mechanisms of posttranscriptional regulation by microRNAs: are the answers in sight? Nat Rev Genet. 2008; 9:102-114. [PubMed: 18197166]

6. Bak M, Silahtaroglu A, Moller M, Christensen M, et al. MicroRNA expression in the adult mouse central nervous system. RNA. 2008; 14:432-444. [PubMed: 18230762] 
7. Smalheiser NR, Lugli G. microRNA regulation of synaptic plasticity. Neuromolecular Med. 2009; 11:133-140. [PubMed: 19458942]

8. Nelson PT, Wang WX, Rajeev BW. MicroRNAs (miRNAs) in neurodegenerative diseases. Brain Pathol. 2008; 18:130-138. [PubMed: 18226108]

9. Turner JD, Williamson R, Almefty KK, Nakaji P, et al. The many roles of microRNAs in brain tumor biology. Neurosurg Focus. 2010; 28:E3. [PubMed: 20043718]

10. Liu DZ, Tian Y, Ander BP, Xu H, et al. Brain and blood microRNA expression profiling of ischemic stroke, intracerebral hemorrhage, and kainate seizures. J Cereb Blood Flow Metab. 2010; 30:92-101. [PubMed: 19724284]

11. Lusardi TA, Farr CD, Faulkner CL, Pignataro G, et al. Ischemic preconditioning regulates expression of microRNAs and a predicted target, MeCP2, in mouse cortex. J Cereb Blood Flow Metab. 2010; 30:744-756. [PubMed: 20010955]

12. Dharap A, Bowen K, Place R, Li LC, et al. Transient focal ischemia induces extensive temporal changes in rat cerebral microRNAome. J Cereb Blood Flow Metab. 2009; 29:675-687. [PubMed: 19142192]

13. Jeyaseelan K, Herath WB, Armugam A. MicroRNAs as therapeutic targets in human diseases. Expert Opin Ther Targets. 2007; 11:1119-1129. [PubMed: 17665982]

14. Jeyaseelan K, Lim KY, Armugam A. MicroRNA expression in the blood and brain of rats subjected to transient focal ischemia by middle cerebral artery occlusion. Stroke. 2008; 39:959966. [PubMed: 18258830]

15. Friedman B, Schachtrup C, Tsai PS, Shih AY, et al. Acute vascular disruption and aquaporin 4 loss after stroke. Stroke. 2009; 40:2182-2190. [PubMed: 19372455]

16. Copin JC, Goodyear MC, Gidday JM, Shah AR, et al. Role of matrix metalloproteinases in apoptosis after transient focal cerebral ischemia in rats and mice. Eur J Neurosci. 2005; 22:15971608. [PubMed: 16197500]

17. Yin KJ, Deng Z, Huang H, Hamblin M, et al. miR-497 regulates neuronal death in mouse brain after transient focal cerebral ischemia. Neurobiol Dis. 2010; 38:17-26. [PubMed: 20053374]

18. Shen J, Yang X, Xie B, Chen Y, et al. MicroRNAs regulate ocular neovascularization. Mol Ther. 2008; 16:1208-1216. [PubMed: 18500251]

19. Tan KS, Armugam A, Sepramaniam S, Lim KY, et al. Expression profile of MicroRNAs in young stroke patients. PLoS One. 2009; 4:e7689. [PubMed: 19888324]

20. Redell JB, Liu Y, Dash PK. Traumatic brain injury alters expression of hippocampal microRNAs: potential regulators of multiple pathophysiological processes. J Neurosci Res. 2009; 87:14351448. [PubMed: 19021292]

21. Lei P, Li Y, Chen X, Yang S, et al. Microarray based analysis of microRNA expression in rat cerebral cortex after traumatic brain injury. Brain Res. 2009; 1284:191-201. [PubMed: 19501075]

22. Sahni V, Mukhopadhyay A, Tysseling V, Hebert A, et al. BMPR1a and BMPR1b signaling exert opposing effects on gliosis after spinal cord injury. J Neurosci. 2010; 30:1839-1855. [PubMed: 20130193]

23. Sofroniew MV. Molecular dissection of reactive astrogliosis and glial scar formation. Trends Neurosci. 2009; 32:638-647. [PubMed: 19782411]

24. Wang WX, Wilfred BR, Madathil SK, Tang G, et al. MiR-107 regulates Granulin/Progranulin with implications for traumatic brain injury and neurodegenerative diseases. Am J Pathol. 2010; 177:334-345. [PubMed: 20489155]

25. He Z, Ong CH, Halper J, Bateman A. Progranulin is a mediator of the wound response. Nat Med. 2003; 9:225-229. [PubMed: 12524533]

26. Yamakuchi M, Lotterman CD, Bao C, Hruban RH, et al. P53-induced microRNA-107 inhibits HIF-1 and tumor angiogenesis. Proc Natl Acad Sci USA. 2010; 107:6334-6339. [PubMed: 20308559]

27. Wang XH, Qian RZ, Zhang W, Chen SF, et al. MicroRNA-320 expression in myocardial microvascular endothelial cells and its relationship with insulin-like growth factor- 1 in type 2 diabetic rats. Clin Exp Pharmacol Physiol. 2009; 36:181-188. [PubMed: 18986336] 
28. Hicks R, Soares H, Smith D, McIntosh T. Temporal and spatial characterization of neuronal injury following lateral fluid-percussion brain injury in the rat. Acta Neuropathol. 1996; 91:236-246. [PubMed: 8834535]

29. Saatman KE, Feeko KJ, Pape RL, Raghupathi R. Differential behavioral and histopathological responses to graded cortical impact injury in mice. J Neurotrauma. 2006; 23:1241-1253. [PubMed: 16928182]

30. Schneider G, Fries P, Wagner-Jochem D, Thome D, et al. Pathophysiological changes after traumatic brain injury: comparison of two experimental animal models by means of MRI. MAGMA. 2002; A14:233-241. [PubMed: 12098566]

31. Kovesdi E, Luckl J, Bukovics P, Farkas O, et al. Update on protein biomarkers in traumatic brain injury with emphasis on clinical use in adults and pediatrics. Acta Neurochir (Wien). 2010; 152:117. [PubMed: 19652904]

32. Liu NK, Wang XF, Lu QB, Xu XM. Altered microRNA expression following traumatic spinal cord injury. Exp Neurol. 2009; 219:424-429. [PubMed: 19576215]

33. Nakanishi K, Nakasa T, Tanaka N, Ishikawa M, et al. Responses of microRNAs 124a and 223 following spinal cord injury in mice. Spinal Cord. 2010; 48:192-196. [PubMed: 19621023]

34. Kiraly M, Kiraly SJ. Traumatic brain injury and delayed sequelae: a review - traumatic brain injury and mild traumatic brain injury (concussion) are precursors to later-onset brain disorders, including early-onset dementia. Scientific World Journal. 2007; 7:1768-1776. [PubMed: 18040539]

35. Stozicka Z, Zilka N, Novak M. Risk and protective factors for sporadic Alzheimer's disease. Acta Virol. 2007; 51:205-222. [PubMed: 18197729]

36. Harold D, Abraham R, Hollingworth P, Sims R, et al. Genome-wide association study identifies variants at CLU and PICALM associated with Alzheimer's disease. Nat Genet. 2009; 41:10881093. [PubMed: 19734902]

37. Jawaid A, Rademakers R, Kass JS, Kalkonde Y, et al. Traumatic brain injury may increase the risk for frontotemporal dementia through reduced progranulin. Neurodegener Dis. 2009; 6:219-220. [PubMed: 20145419]

38. Zhang X, Le W. Pathological role of hypoxia in Alzheimer's disease. Exp Neurol. 2010; 223:299_ 303. [PubMed: 19679125]

39. Smith DH, Uryu K, Saatman KE, Trojanowski JQ, et al. Protein accumulation in traumatic brain injury. Neuromolecular Med. 2003; 4:59-72. [PubMed: 14528053]

40. Rovelet-Lecrux A, Hannequin D, Raux G, Le Meur N, et al. APP locus duplication causes autosomal dominant early-onset Alzheimer disease with cerebral amyloid angiopathy. Nat Genet. 2006; 38:24-26. [PubMed: 16369530]

41. Uryu K, Giasson BI, Longhi L, Martinez D, et al. Age-dependent synuclein pathology following traumatic brain injury in mice. Exp Neurol. 2003; 184:214-224. [PubMed: 14637093]

42. Pinkston JB, Alekseeva N, Gonzalez Toledo E. Stroke and dementia. Neurol Res. 2009; 31:824831. [PubMed: 19723451]

43. Guglielmotto M, Tamagno E, Danni O. Oxidative stress and hypoxia contribute to Alzheimer's disease pathogenesis: two sides of the same coin. Scientific WorldJournal. 2009; 9:781-791. [PubMed: 19705038]

44. McKee AC, Gavett BE, Stern RA, Nowinski CJ, et al. TDP-43 proteinopathy and motor neuron disease in chronic traumatic encephalopathy. J Neuropathol Exp Neurol. 2010; 69:918-929. [PubMed: 20720505]

45. Wang WX, Rajeev BW, Stromberg AJ, Ren N, et al. The expression of microRNA miR-107 decreases early in Alzheimer's disease and may accelerate disease progression through regulation of beta-site amyloid precursor protein-cleaving enzyme 1. J Neurosci. 2008; 28:1213-1223. [PubMed: 18234899]

46. Nelson PT, Wang WX. MiR-107 is reduced in Alzheimer's disease brain neocortex: validation study. J Alzheimers Dis. 2010; 21:75-79. [PubMed: 20413881]

47. Blasko I, Beer R, Bigl M, Apelt J, et al. Experimental traumatic brain injury in rats stimulates the expression, production and activity of Alzheimer's disease beta-secretase (BACE-1). J Neural Transm. 2004; 111:523-536. [PubMed: 15057522] 
48. Uryu K, Chen XH, Martinez D, Browne KD, et al. Multiple proteins implicated in neurodegenerative diseases accumulate in axons after brain trauma in humans. Exp Neurol. 2007; 208:185-192. [PubMed: 17826768]

49. Shaked I, Meerson A, Wolf Y, Avni R, et al. MicroRNA-132 potentiates cholinergic antiinflammatory signaling by targeting acetylcholinesterase. Immunity. 2009; 31:965-973. [PubMed: 20005135]

50. Zhang J, Guo H, Qian G, Ge S, et al. MiR-145, a new regulator of the DNA fragmentation factor-45 (DFF45)-mediated apoptotic network. Mol Cancer. 2010; 9:211. [PubMed: 20687965] 


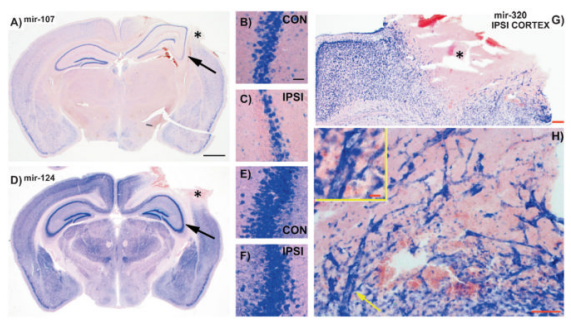

Figure 1.

In situ hybridization for miR-107, -124, and -320 in a brain-injured mouse 24 hours after cortical impact. MiR-107, -124 , and -320 are expressed throughout the mouse brain. Controlled cortical impact injury $(n=5)$ and in situ hybridizations were performed according to our previously published paper [24]. A: Reduced miR-107 in the cortex on the side of the impact $(*)$ compared to the contralateral side. B, C: High magnification images of contralateral and ipsilateral (arrow) CA-3, respectively, for miR-107 staining. D: Reduced miR-124 expression in the contused cortex (*). E, F: High magnification images of CA-3 showing milder loss in staining intensity in ipsilateral CA-3 compared to miR-107. G, H: MiR-320 staining in the ipsilateral (contused) cortex at low and high magnifications, respectively. MiR-320 expression was dramatically reduced in the contused cortex (*); however, the tissue bordering the contusion core showed marked up-regulation in miR-320 in blood vessels. $\mathbf{H}$ : High magnification image of many blood vessels positive for miR-320 (inset in $\mathbf{H}$ : magnified photo of a blood vessel noted by arrow). Scale A, D = 500 $\mu \mathrm{m} ;(\mathbf{B})$, $(\mathbf{C}),(\mathbf{E}),(\mathbf{F})=50 \mu \mathrm{m} ;(\mathbf{G})=100 \mu \mathrm{m} ;(\mathbf{H})=100 \mu \mathrm{m}$ and inset $=10 \mu \mathrm{m}$. CON: contralateral, IPSI: ipsilateral. 


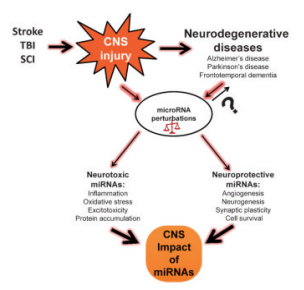

Figure 2.

miRNA involvement in acute CNS injury and neurodegeneration. Schematic diagram depicting how acute CNS injury may contribute to neurodegenerative disease progression through altered miRNAs. Experimental evidence suggest that stroke, TBI and SCI drive the brain towards progressive neurodegeneration. Research has also identified altered miRNA profiles following CNS injury and in neurodegenerative diseases. We predict that the connecting link between CNS injury and neurodegenerative diseases is altered/aberrant miRNA expression (no-glow arrow, marked by “?”). Following CNS injury, many miRNAs are altered, precipitating a spectrum of mRNA changes that affect multiple signaling pathways. These amendments in miRNA expression can either be neuroprotective or detrimental. Therapeutic strategies aiming to promote the effects of neuroprotective miRNAs or to reduce the effects of neurotoxic miRNAs may be able to halt the progression of injury pathology. TBI, traumatic brain injury; SCI, spinal cord injury. 
Table 1

Brain microRNAs dysregulated in CNS injury

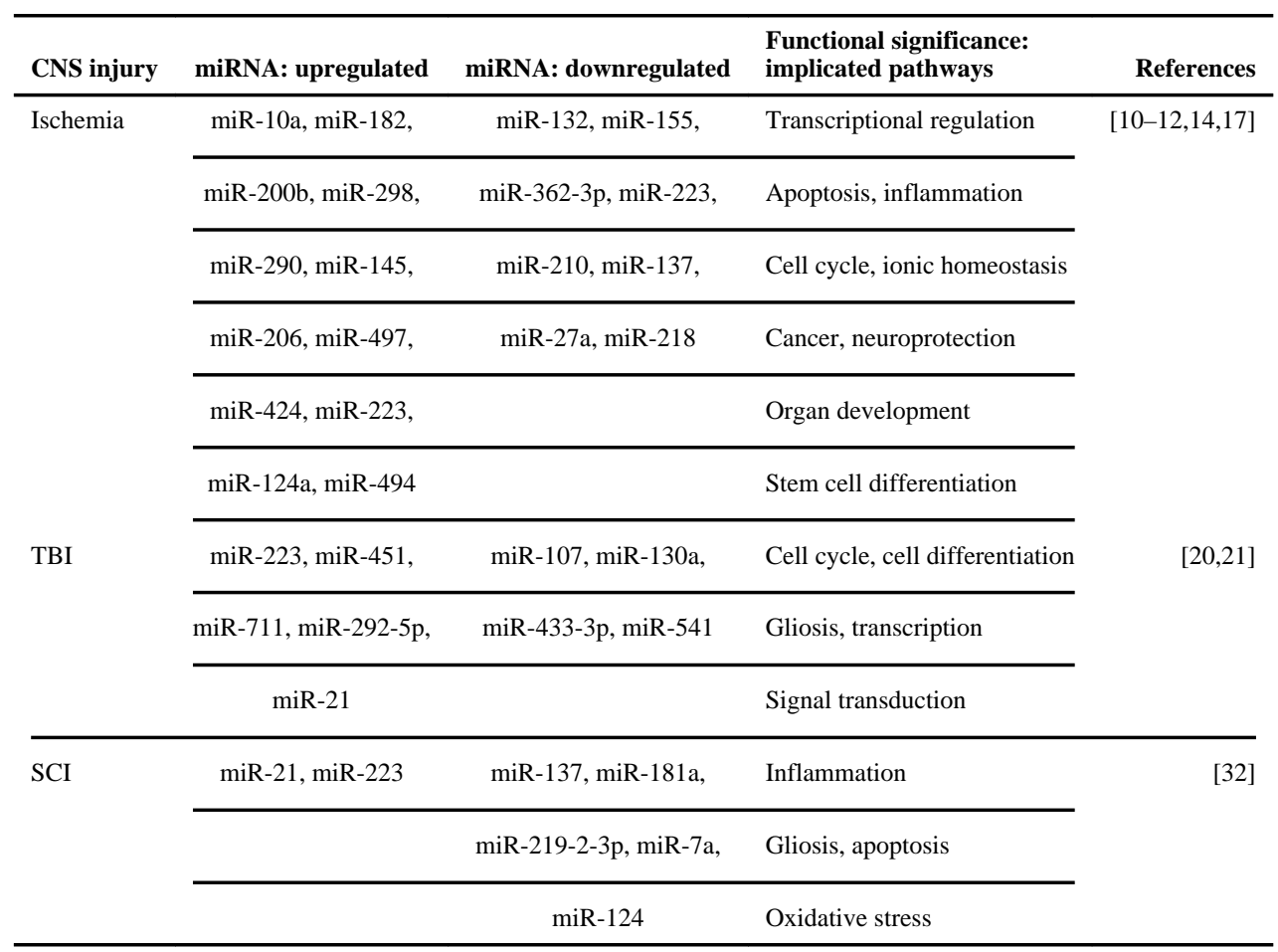

Abbreviations: CNS, central nervous system; miRNA, microRNA; TBI, traumatic brain injury; SCI, spinal cord injury.

Following CNS injury, several miRNAs show altered expression in microarray profiling. The table contains only those profiles that are validated by RT-PCR or in situ staining. 